\title{
Differences in growth and alkaline phosphatase activity between Microcystis aeruginosa and Chlorella pyrenoidosa in response to media with different organic phosphorus
}

\author{
Xiaoli SHI*, Shanqin QIAN, Fanxiang KONG, Min ZHANG and Yang YU \\ State Key Laboratory of Lake Science and Environment, Nanjing Institute of Geography and Limnology, Chinese Academy of \\ Sciences, 73 Beijing East Road, Nanjing 210008, P.R. China \\ *e-mail corresponding author: xlshi@niglas.ac.cn
}

\begin{abstract}
The growth of Microcystis aeruginosa and Chlorella pyrenoidosa in three dissolved organic phosphorus sources (glucose-1phosphate, adenosine triphosphate, cyclic-adenosine monophosphate) were studied in cultures separated by a dialysis membrane. Results showed that M. aeruginosa and C. pyrenoidosa could utilize those three forms of organic phosphorus, but their growth rates and cell abundances were low in comparison with those in the orthophosphate control. M. aeruginosa had a higher growth rate than C. pyrenoidosa in glucose-1-phosphate, and then became dominate in the separate cultures. In contrast, those two algal species didn't show any significant differences in the growth rate and cell abundance in the medium with adenosine triphosphate and cyclicadenosine monophosphate. Alkaline phosphatase was an important enzyme for hydrolyzing glucose-1-phosphate, adenosine triphosphate and cyclic-adenosine monophosphate, the activity of which was positively correlated with the growth rate of algae. Considering the big proportion of glucose-1-phosphate in the Lake Taihu, the capability of M. aeruginosa to efficiently utilize this type of organic phosphorus source might be one of reason that why M. aeruginosa is the dominant species in this hyper-eutrophic lake.
\end{abstract}

Key words: Microcystis aeruginosa, Chlorella pyrenoidosa, organic phosphorus, alkaline phophatase, Lake Taihu

\section{INTRODUCTION}

Phosphorus is frequently the limiting nutrient for phytoplankton growth in freshwater ecosystems (Schindler 1977; Hecky \& Kilham 1988; Hudson et al. 2000), and its availability is an important factor to control phytoplankton productivity and species composition in lakes (Cotner \& Wetzel 1992). Eutrophication is one of the most widespread environmental problems of inland waters. Concomitant with the process of eutrophication, the proportion of dissolved organic phosphorus in the total phosphorus pool has been dramatically increased (Hudson et al. 2000). For instance, soluble phosphorus comprises as high as $88 \%$ of dissolved organic phosphorus in the Lake Taihu, a hyper-eutrophic shallow lake, in summer. Therefore, when external inorganic phosphorus concentration is very low in the water column during summer algal blooms, the extent to which algae could benefit from organic phosphorus compounds may be one of key factors to determine the algal interspecific competition.

Some algae are capable of obtaining phosphate from dissolved organic phosphorus (DOP) in the absence of dissolved inorganic phosphorus (DIP) to sustain their growth (Whitton et al. 1991; Oh et al. 2002). The utilization of DOP is associated with a number of enzymes, among which alkaline phosphatase was regarded as the most important one in hydrolyzing a variety of forms of DOP sources (Shan et al. 1994). Alkaline phosphatase activity (APA) is often a common marker of phosphate stress in many phytoplankton (Dyhrman \& Palenik 1999), and the phosphorus status of phytoplankton communities can be evaluated by measurement of total APA in enzyme assays (Nicholson et al. 2006).

Microcystis are widely present as the dominant species in the eutrophic freshwater lake. Is Microcystis capable of utilizing organic phosphorus more efficiently than other algae? In our previous work, we investigated the effect of inorganic phosphate (pyrophosphate, polyphosphate) on the competition of M. aeruginosa and $C$. pyrenoidosa (Qian et al. 2008). Here in current research, our objective is to study the growth of $M$. aeruginosa and $C$. pyrenoidosa under three types of organic phosphorus, glucose-1-phosphate, adenosine triphosphate (ATP), cyclic-adenosine monophosphate (c-AMP), which were widely present in the freshwater ecosystem. Meanwhile we estimated cellular alkaline phosphatase activity to study its relationship with the capability of phytoplankton to utilize different forms of DOP compounds.

\section{METHODS}

\subsection{Organisms and cultivation}

M. aeruginosa and C. pyrenoidosa used in our experiment were obtained from the Institute of Hydrobiology of the Chinese Academy of Sciences. The algae were grown in batch culture in BG-11 medium at $25^{\circ} \mathrm{C}$ 
Tab. 1 The maximum growth rate $\left(\mu\right.$ day $\left.^{-1}\right)$ of $M$. aeruginosa and $C$. pyrenoidosa under different DOP treatments.

\begin{tabular}{lcccc}
\hline & $\mathrm{KH}_{2} \mathrm{PO}_{4}$ & glucose-1-phosphate & ATP & c-AMP \\
\hline M. aeruginosa & 0.32 & 0.25 & 0.18 & 0.15 \\
C. pyrenoidosa & 0.41 & 0.19 & 0.15 & 0.18 \\
\hline
\end{tabular}

under fluorescence light at an intensity of $40 \mu \mathrm{E} \mathrm{m}^{-2} \mathrm{~s}^{-1}$ with a 12:12h light/dark cycle. All of chemical reagents were AP grade and made in China.

\subsection{Experimental design}

The experiment was performed with cultures separated by a dialysis membrane, which had a molecular weight cut-off of 12-14 kDa (Cole-Parmer Instrument Co., USA), as described by Hyenstrand et al. (2000). M. aeruginosa and C. pyrenoidosa were physically separated but shared the same nutrient conditions (Qian et al. 2008). Glucose-1-phosphate, adenosine triphosphate and cyclic adenosine monophosphate were chosen as organic phosphorus sources, meanwhile, inorganic phosphate treatment was made as a control. The concentration of the all types of phosphorus was $2.72 \mathrm{mg} P$ $\mathrm{L}^{-1}$, which is the half level of phosphorus for BG-11 medium. All of treatments were in triplicate. Cells of $M$. aeruginosa and C. pyrenoidosa during the log growth phase were inoculated into the sterile medium with different organic phosphorus treatments, at an initial cell abundance of $6.5 \times 10^{4}$ cells $\mathrm{mL}^{-1}$.

\subsection{Analysis}

Algae were filtered out and the Total Dissolved Phosphorus (TDP) concentration of the medium was measured with the molybdenum blue colorimetric method (Huang et al. 2000) after digestion with $\mathrm{K}_{2} \mathrm{~S}_{2} \mathrm{O}_{8}$ at $121{ }^{\circ} \mathrm{C}$ for $30 \mathrm{~min}$. The activity of cellular alkaline phosphatase of algae was determined according to Berman (1970) and Nicholson et al. (2006). Algae were enumerated under microscope in a counting chamber. All the parameters were assayed every two days.

The data were analyzed with Origin 8.0, and significant differences $(p<0.05)$ were evaluated with one-way analysis of variance.

\section{RESULTS}

M. aeruginosa and $C$. pyrenoidosa had the capability to utilize those three forms of organic phosphorus, and both of them had an obvious growth. However, their growth rate with organic phosphorus was much lower than that with orthophosphate (Tab. 1). Comparing the growth rate under different types of organic phosphorus, $M$. aeruginosa grew the best in glucose-1-phosphate, with the maximum cell abundance of $1.80 \times 10^{6}$ cells $\mathrm{mL}^{-1}$. ATP was the second best organic phosphorus source for M. aeruginosa, with its maximum cell abundance reaching to $1.39 \times 10^{6}$ cells $\mathrm{mL}^{-1}$. The last utilizable one is c-AMP, but there was no significant differ- ence for the later two organic phosphorus sources ( $p$ $>0.05$ ) (Fig. 1). C. pyrenoidosa showed the equivalent ability to utilize those three types of organic phosphorus, but the maximum cell abundances were lower than those of $M$. aeruginosa, being $1.26 \times 10^{6}$ cells $\mathrm{mL}^{-1}$, $1.30 \times 10^{6}$ cells mL $\mathrm{mL}^{-1}$ and $1.21 \times 10^{6}$ cells $\mathrm{mL}^{-1}$ with glucose-1-phosphate, ATP and c-AMP, respectively (Fig. 1). Under glucose-1-phosphate condition, M. aeruginosa had a longer lag stage than $C$. pyrenoidosa, but a sharp growth after day 6 , and eventually $M$. aeruginosa became dominant over $C$. pyrenoidosa in the separate cultures. In contrast, those two algal species did not show any significant differences in the maximum growth rates and cell abundances in ATP and c-AMP medium during the experimental period $(p>0.05)$. However, C. pyrenoidosa grew better than M. aeruginosa in orthophosphate medium during whole experimental process (Fig. 1). The variation of phosphorus concentration in water was closely related to the growth of algae, with the declining rate increasing when the algal growth rate became higher (Fig. 1). The cell abundance of $M$. aeruginosa and C. pyrenoidosa were relatively high in the orthophosphate than in DOP medium, thus, the consumption of phosphorus were bigger than that in glucose-1-phophate, ATP and cAMP medium (Fig. 1).

Cellular APA of M. aeruginosa increased at the beginning, reaching the maximum level at day 4 , and then decreased constantly until the end of the experiment (Fig. 2). Whereas, cellular APA activity of $C$. pyrenoidosa showed a continuous decline, and became stable after day 6 , without any significant differences between DOP treatments $(p>0.05)$. The maximum value of APA activity appeared higher in the glucose-1phosphate medium than in the medium of ATP and cAMP for M. aeruginosa, and it had no significant difference for the treatment of ATP and c-AMP (Fig. 2).

\section{DISCUSSION}

\subsection{The relationship between the alkaline phosphatase activity and the capability to utilize organic phosphorus}

Alkaline phosphatase, which can be released from bacteria and phytoplankton in both marine and freshwater, can catalyze the liberation of orthophosphate from varying phosphorus compounds, e.g. phosphate monoesters, nucleotide polyphosphate, deoxynucleotide polyphosphate, inorganic pyrophosphate, tripolyphosphate, and some S-phosphrothioate esters (Shan et al. 1994). Phosphatase enzymes are mainly located at the periphery of algal and bacterial cells but also occur free 


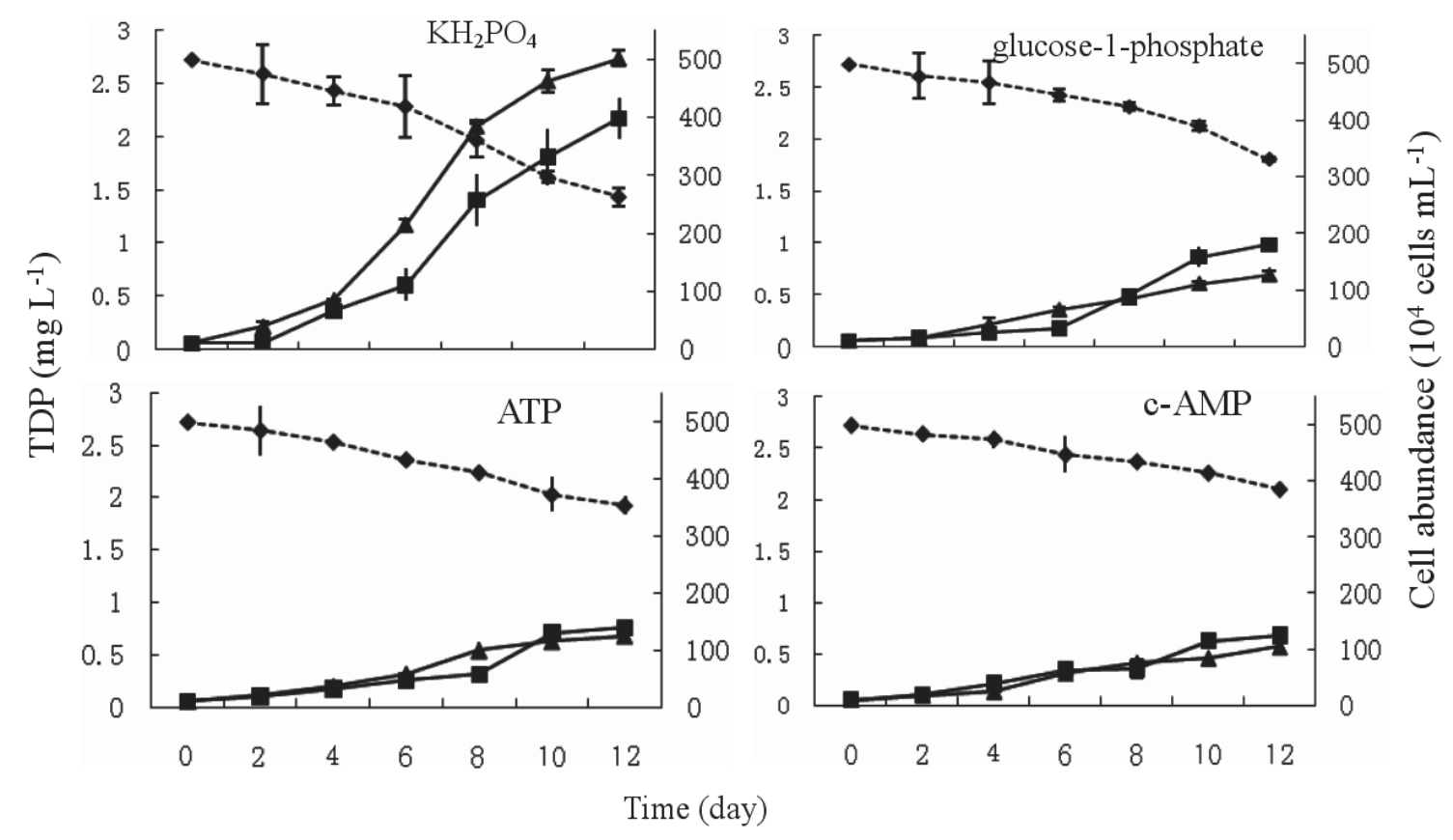

Fig. 1. Cell abundance of M. aeruginosa and C. pyrenoidosa and total dissolved phosphorus concentration in medium under separate batch cultures. $M$. aeruginosa; $\boldsymbol{\Delta}$ C. pyrenoidosa. Solid line represents cell abundance and dashed line represents total dissolved phosphorus concentration in the medium. Symbols and error bars represent average and standard deviation of replicate cultures.

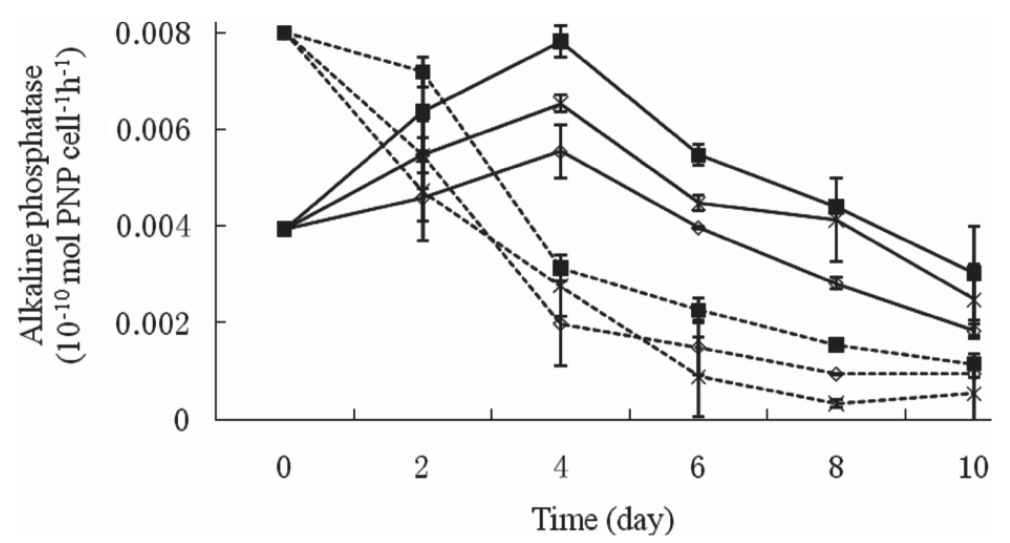

Fig. 2. Cellular alkaline phosphatase activity of $M$. aeruginosa and C. pyrenoidosa. glucose-1-phosphate; $\times$ ATP; $\diamond \mathrm{c}-\mathrm{AMP}$. Solid line represents $M$. aeruginosa, and dashed line represents $C$. pyrenoidosa. Symbols and error bars represent average and standard deviation of replicate cultures.

dissolved in the water column (Spijkerman \& Coesel 1998). Its activity was widely regarded as an indicator to access the degree of $\mathrm{P}$ limitation in an aquatic ecosystem or a single species, and also provides information about the utilization of DOP as an additional source of phosphate by bacteria and primary producers.

Our study showed that the variation trend of APA for $M$. aeruginosa and C. pyrenoidosa were similar under DOP and phosphate (pyrophosphate and polyphosphate) treatments, but its values were relatively higher for DOP than for phosphate (Qian et al. 2008).
As alkaline phosphatase is often used as a metric of DOP bioavailability (Dyhrman \& Ruttenberg 2006), this indicated that glucose-1-phosphate, adenosine triphosphate, adenosine monophosphate are more bioavailable than pyrophosphate and polyphosphate. In addition, cellular phosphatase activity was well related to the growth of algae under DOP treatments, with the higher enzyme activity the more rapid algal growth rate. The relatively high maximum value of APA of $M$. aeruginosa in glucose-1-phosphate media implied that glucose-1-phosphate is more readily used by $M$. aeruginosa than other two DOP compounds. 


\subsection{Algal growth under different forms of organic phosphorus and its ecological meaning}

Dissolved organic phosphorus comprises a significant proportion of the total dissolved phosphorus compounds. However, this fraction is poorly characterized largely due to the diversity and complexity of the compounds present (Shan et al. 1994). In our experiment, we selected three types of organic phosphorus, which are generally present in the eutrophic water body. Dissolved c-AMP could have resulted from the different combined release of cAMP by both pelagic and littoral organisms, as well as from sediments and allochthonous sources, which often comprised $1-5 \%$ of the total dissolved phosphorus in surface waters (Francko \& Wetzel 1982). Meanwhile, besides phosphorus source, cAMP was reported as an effective signal compound, leading to significant increase of cultivation efficiency of bacterioplankon (Bruns et al. 2003). Dissolved ATP was another selected organic phosphorus in our research, which has been reported to occur in a variety of aquatic habitats and plays integral role in the regulation of metabolic activities in all living cells. Phosphomonoester was detected to exist with significant quantities in lake water (Francko \& Heath 1979) and ocean (Taft et al. 1977; Karl \& Yanagi 1997), which was also reported as an important component of the organic phosphorus in the sediment of the hyper-eutrophic lake (Lake Taihu), where the release from sediments are the major sources of phosphorus loading to the water column (Bai et al. 2009).

Our results indicated that $M$. aeruginosa and $C$. pyrenoidosa were able to utilize those three typical forms of organic phosphorus, but orthophosphate is the preferred source. Among those three organic phosphorus, $M$. aeruginosa utilized glucose-1-phosphate preferentially than ATP and c-AMP, with no significant difference for the later two. While C. pyrenoidosa showed the equivalent utilizable ability for those three organic phosphorus sources, since its maximum cell abundance and alkaline phosphatase activity didn't show significant difference under all those treatments.

Our previous study indicated that $C$. pyrenoidosa had a higher growth rate and greater ability to utilize pyrophosphate and polyphosphate than $M$. aeruginosa in the unialgal culture, while in the separated culture $C$. pyrenoidosa underperformed $M$. aeruginosa probably due to allelopathy effects (Qian et al. 2008). In contrast, this study revealed that $M$. aeruginosa could compete over $C$. pyrenoidosa in glucose-1-phosphate treatment in the separated culture, which is also true in the unialgal culture (Qian et al. 2010), implying that M. aeruginosa is able to more efficiently utilize this type of organic phosphorus than C. pyrenoidosa. However, no obvious difference could be observed for those two algal species when grew in ATP and c-AMP medium.

\section{CONCLUSIONS}

Two main conclusions could be drawn from this study. First, because of the growth of algae differs depending on the component of phosphorus source, algal assemblage could be largely decided by the composition of phosphorous source in the phosphorus-limiting water ecosystem. Second, the relatively strong ability for M. aeruginosa to utilize glucose-1-phosphate may be one of reason that why $M$. aeruginosa is the dominant species in eutrophic Lake Taihu, where glucose-1-phosphate was reported as an important component of the organic phosphorus in the sediment of the hyper-eutrophic lake, where the release from sediments are the major sources of P loading to the water column.

\section{ACKNOWLEDGEMENTS}

This work were granted by National Basic Research Program of China '973' (No. 2008CB418005) and the State Natural Science Foundation of China (No. 31070420; No. 40601034).

\section{REFERENCES}

Ammerman, J.W. \& F. Azam. 1985. Bacterial 5-Nucleotidase in aquatic ecosystems: a novel mechanism of phosphorus regeneration. Science, 227: 1338-1440.

Bai, X., S. Ding, C. Fan, T. Liu, D. Shi \& L. Zhang. 2009. Organic phosphorus species in surface sediments of la large, shallow, eutrophic lake, Lake Taihu, China. Envion. Pollut., 157: 2507-2513.

Berman, T. 1970. Alkaline phosphatases and phosphorus availability in Lake Kinneret. Limnol. Oceanogr., 15: 663-674.

Bruns, A., U. Nübel, H. Cypionka \& J. Overmann. 2003. Effect of signal compounds and incubation conditions on the culturability of freshwater bacterioplankton. Appl. Environ. Microbiol., 69: 1980-1989.

Cotner, J.B. \& R.G. Wetzel. 1992. Uptake of dissolved inorganic and organic compounds by phytoplankton and bacterioplankton. Limnol. Oceanogr., 37: 232-243.

Dyhrman S.T. \& B. Palenik. 1999. Phosphate stress in cultures and field populations of dinofalegellate Prorocentrum minimum detected by a single-cell alkaline phosphatase assay. Appl. Environ. Microbiol., 65: 3205-3212.

Dyhrman, S.T. \& K.C. Ruttenberg. 2006. Presence and regulation of alkaline phosphatase activity in eukaryotic phytoplankton from the coastal ocean: implications for dissolved organic phosphorus remineralization. Limnol. Oceanogr., 51: 1381-1390.

Francko, D.A. \& R.T. Heath. 1979. Functionally distinct classes of complex phosphorus compounds in lake water. Limnol. Oceanogr., 24: 463-473.

Francko, D.A. \& R.G. Wetzel. 1982. The isolation of cyclic adenosine 3'5'-monophosphate (cAMP) from lakes of differing trophic status: Correlation with planktonic metabolic variables. Limnol. Oceanogr., 27: 27-38.

Hecky, R.E. \& P. Kilham. 1988. Nutrient limitation of phytoplankton in freshwater and marine environments: A review of recent evidence on the effects of enrichment. Limnol. Oceanogr., 33: 796-822.

Huang, X., W. Chen \& M. Cai. 2000. Survey, observation and analysis of lake ecology. Standards Press of China, Beijing: $198 \mathrm{pp}$.

Hudson, J.J., W.D. Taylor \& D.W. Schindler. 2000. Phosphate concentrations in lakes. Nature, 406: 54-56. 
Hyenstrand, P., U.A. Burkert, A. Petterson \& P. Blomqvist. 2000. Competition between the green alga Scenedesmus and the cyanobacterium Synechococcus under different modes of inorganic nitrogen supply. Hydrobiology, 435: 91-98.

Karl, D.M. \& K. Yanagi. 1997. Partial characterization of the dissolved organic phosphorus pool in the oligotrophic North Pacific Ocean. Limnol. Oceanogr., 42: 1398-1405.

Nicholson, D., S. Dyhrman, F. Chavea \& A. Paytan. 2006. Alkaline phosphatase activity in the phytoplankton communities of Monterey Bay and San Francisco Bay. Limnol. Oceanogr., 51: 874-883.

Oh, S.J., T. Yamamoto, Y. Kataoka, O. Matsuda, Y. Matsuyama \& Y. Kotani. 2002. Utilization of dissolved organic phosphorus by the two toxic dinoflagellates, Alexandrium tamarense and Gymnodinium catenatum (Dinophyceae). Fisheries Science, 68: 416-424.

Qian, S.Q., F.X. Kong, X.L. Shi, M. Zhang, X. Tan \& Z. Yang. 2008. Interspecific interaction between Microcystis aeruginosa and Chlorella pyrenoidosa in different phosphate media. J. Freshwat. Ecol., 23: 635-642.

Received: June 2010

Accepted: October 2010
Qian, S.Q., F.X. Kong, M. Zhang, Y. Yu \& X.L. Shi. 2010. Utilization of dissolved organic phosphorus and the growth of Microcystis aeruginosa and Chlorella pyrenoidosa in the uniagal culture. J. Lake Sci., 22: 411-415 (in Chinese).

Schindler, D.W. 1977. Evolution of phosphorus limitation in lakes. Science, 195: 260-262.

Shan, Y., I.D. McKelvie \& B.T. Hart. 1994. Determination of alkaline phosphatase-hydrolyzable phosphorus in natural water systems by enzymatic flow injection. Limnol. Oceanogr., 39: 1993-2000.

Spijkerman, E. \& P.F.M. Coesel. 1998. Alkaline phosphatase activity in two planktonic desmid species and the possible role of an extracellular envelop. Freshwat. Biol., 39: 503-513.

Taft, J.L., M.E. Loftus \& W.R. Taylor. 1977. Phosphate uptake from phosphomonoesters by phytoplankton in the Chesapeake Bay. Limnol. Oceanogr., 22: 1012-1021.

Whitton, B.A., S.L.J. Grainger, G.R.W. Hawley \& J.W. Simon. 1991. Cell-bound and extracellular activities of Cyanobacterial isolates. Microbial Ecol., 21: 85-98. 